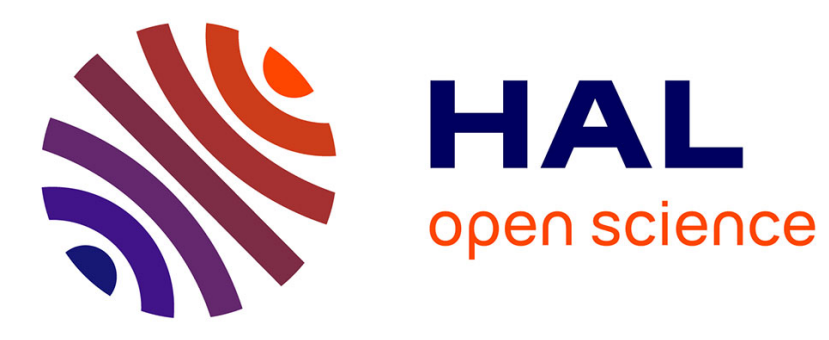

\title{
Ultrasound-Assisted Liquid-Phase Synthesis and Mechanical Properties of Aluminum Matrix Nanocomposites Incorporating Boride Nanocrystals
}

Binghua Ma, Isabel Gómez-Recio, L. Mazerolles, Pierre-emmanuel Mazeran, Clément Sanchez, Stéphane Delalande, David Portehault

\section{To cite this version:}

Binghua Ma, Isabel Gómez-Recio, L. Mazerolles, Pierre-emmanuel Mazeran, Clément Sanchez, et al.. Ultrasound-Assisted Liquid-Phase Synthesis and Mechanical Properties of Aluminum Matrix Nanocomposites Incorporating Boride Nanocrystals. Small, 2022, 18, pp.2104091. 10.1002/smll.202104091 . hal-03428746

\section{HAL Id: hal-03428746 https://hal.science/hal-03428746}

Submitted on 20 Nov 2021

HAL is a multi-disciplinary open access archive for the deposit and dissemination of scientific research documents, whether they are published or not. The documents may come from teaching and research institutions in France or abroad, or from public or private research centers.
L'archive ouverte pluridisciplinaire HAL, est destinée au dépôt et à la diffusion de documents scientifiques de niveau recherche, publiés ou non, émanant des établissements d'enseignement et de recherche français ou étrangers, des laboratoires publics ou privés. 


\section{Ultrasound-assisted liquid-phase synthesis and}

\section{mechanical properties of aluminum matrix}

\section{nanocomposites incorporating boride nanocrystals}

Binghua Ma,${ }^{1,2}$ Isabel Gómez-Recio, ${ }^{1}$ Leo Mazerolles, ${ }^{3}$ Pierre-Emmanuel Mazeran, ${ }^{4}$ Clement

Sanchez, ${ }^{l}$ Stephane Delalande, ${ }^{2 *}$ David Portehault ${ }^{1 *}$

${ }^{1}$ Sorbonne Université, CNRS, Collège de France, Laboratoire de Chimie de la Matière

Condensée de Paris (LCMCP), 4 place Jussieu, F-75005, Paris, France

${ }^{2}$ Stellantis, Centre Technique de Vélizy, route de Gisy, Vélizy-Villacoublay, 78140, France

${ }^{3}$ Institut de Chimie et des Matériaux Paris-Est, UMR 7182, Université Paris Est, CNRS, 2-8 rue H. Dunant, 94320, Thiais, France

${ }^{4}$ Alliance Sorbonne Université, Université de Technologie de Compiègne, Laboratoire Roberval de Mécanique, FRE UTC-CNRS 2012, Compiègne, France

*Corresponding authors: david.portehault@ sorbonne-universite.fr

stephane.delalande@stellantis.com 


\section{ABSTRACT}

Incorporating boride nanocrystals could significantly impact the mechanical properties of aluminum alloys. Molten salts synthesis offers opportunities to fabricate superhard boride nanoparticles, which can sustain the harsh conditions of liquid metallurgy synthesis, thus enabling liquid-phase design of metallic nanocomposites. Here we unveil $\mathrm{HfB}_{2}-\mathrm{Al}$ nanocomposites with molten salt-derived $\mathrm{HfB}_{2}$ nanoparticles incorporated by ultrasound-assisted casting. Using sequential dilutions, the nanoparticles were incorporated in Al with macroscale homogeneity. The crystal structure and size of the nanocrystals is retained in the final nanocomposites, supporting their excellent chemical stability. Semi-coherent interfaces between the nanoparticles and the matrix are then evidenced by transmission electron microscopy, thus suggesting that the nanocrystals could act as nuclei for the solidification of $\mathrm{Al}$ and then limit the Al grain size to $<$ ca. $20 \mu \mathrm{m}$. Nanoindentation measurements revealed significant grain boundaries strengthening effect. We finally show that $\mathrm{HfB}_{2}$ nanoparticles also enable a strong decrease in matrix grain size and an increase in the hardness of the $\mathrm{AlSi}_{7} \mathrm{Cu}_{0.5} \mathrm{Mg}_{0.3}$ cast alloy. This proof-of-concept material is paving the way to new light-weight Al matrix nanocomposites doped by molten-salt synthesized nanoparticles. 


\section{INTRODUCTION}

Aluminum matrix nanocomposites raise wide attention in multiple fields such as transportation and aerospace sectors due to their light-weight, specific strength, good wear resistance and high stability. ${ }^{1-3}$ Powder metallurgy has been successfully used to fabricate different types of nanocomposites in lab-scale., ${ }^{2,4}$ Researchers have also obtained nanocomposites by foundry metallurgy to ensure large-scale manufacture production. ${ }^{4}$ Efforts are still needed to achieve high mechanical properties with simple process. ${ }^{2,4}$ One of the key challenges in fabricating metal matrix nanocomposites by liquid metallurgy process lies in the harsh conditions of the nanoparticles feeding and dispersion process at high temperature (over solidus or liquidus of aluminum alloys, generally above $550{ }^{\circ} \mathrm{C}$ ). Indeed, the stability of nanoparticles at high temperature is always a concern in these conditions. In the past decades, classic aluminum matrix nanocomposites have relied on commercial submicronic particles with limited choices, including $\mathrm{SiC}, \mathrm{Al}_{2} \mathrm{O}_{3}, \mathrm{TiC}, \mathrm{TiB}_{2}$, etc. ${ }^{5}$ Recent progress in the in situ growth of oxide, boride and carbide nanoparticles has shown some promises to increase nanoparticle loading and to enhance interfacial bonding between nanoparticles and metal matrix. ${ }^{6-8}$ In particular, the reaction of $\mathrm{K}_{2} \mathrm{TiF}_{6}$ and $\mathrm{KBF}_{4}$ in molten mixtures of aluminum and fluoride salts enabled the in situ formation of $\mathrm{TiB}_{2}$ nanoparticles in different aluminum alloys. ${ }^{8}$ However, two main concerns still exist: difficulties to control the particle size and presence of impurities in the final products. ${ }^{7-11}$

The continuous development of nanoparticle synthesis approaches, especially the emergence of molten salts synthesis offers an alternative solution. ${ }^{12}$ Several classes of nanoparticles including borides, nitrides, carbides and silicides have been synthesized in molten 
salt solvents. ${ }^{13}$ The use of inorganic molten salts as high temperature solvents $\left(300\right.$ to $\left.900{ }^{\circ} \mathrm{C}\right)$ could lead to nanoparticles stable at the processing temperature of liquid metallurgy. Assynthesized nanoparticles possess well-controlled particle size, ligand-free and could be easily dispersed in water or alcohols after washing. All these developments taken together, nanoparticles prepared in molten salts appear as interesting potential precursors to create new metal matrix nanocomposites. Nevertheless, there are still several issues to overcome to reach this aim, such as the demixing of nanoparticles powders from the molten matrix during incorporation and solidification.

Among different classes of materials accessible by molten-salts synthesis, borides are hard and promising candidates to further reinforce light-weight alloys as they have exceptional combination of properties like oxidation stability, high hardness, chemical inertness, etc. ${ }^{2}$ Previous research on boride-Al nanocomposites mainly focused on the in situ and ex situ fabricated $\mathrm{TiB}_{2}-\mathrm{Al}$ for enhanced mechanical properties., ${ }^{4,10,14,15} \mathrm{TiB}_{2}$ is a well-known $\mathrm{Al}$ grain growth inhibitor and its grain refinement ability has also been well documented by the study of the orientation relationships. ${ }^{15} \mathrm{HfB}_{2}$ is a superhard material with theoretical hardness above $40 \mathrm{GPa},{ }^{16}$ which is larger than $\mathrm{TiB}_{2}{ }^{17}$ and then supports its study as nanoscaled reinforcement inclusions. $\mathrm{HfB}_{2}$ has been applied to fabricate ceramic composites such as $\mathrm{HfB}_{2}-\mathrm{B}_{4} \mathrm{C}$ or $\mathrm{SiC}$ $\mathrm{HfB}_{2}{ }^{18-20}$ It also contains hafnium, a heavy element, which facilitates transmission electron microscopy studies and chemical detection in the final composites. $\mathrm{HfB}_{2}$ - $\mathrm{Al}$ nanocomposites should then be a useful probe to reach a proof-of-concept material for the incorporation of other molten salts synthesized boride nanoparticles in Al matirx.

In this work, we firstly synthesize $\mathrm{HfB}_{2}-\mathrm{Al}$ nanocomposites by using molten salts as solvent to prepare $\mathrm{HfB}_{2}$ nanocrystals. We then use high temperature ultrasound-assisted casting 
to incorporate the nanoparticles into aluminum-based matrices. (Figure 1) The dispersion behavior of the nanoparticles in molten aluminum was investigated. The study of interface between nanoparticles and the matrix provides hints for nanoparticles-triggered heterogeneous nucleation of the Al crystal grains during solidification, thus limiting the Al-based matrix grain size below $20 \mu \mathrm{m}$, which significantly increase hardness by grain boundaries strengthening.

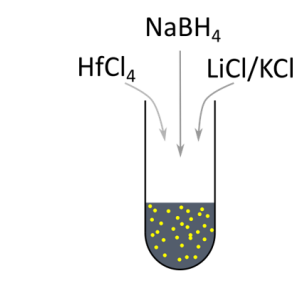

Molten salts synthesis

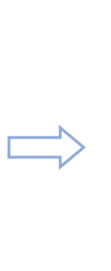

$$
\text { - Al }
$$$$
\text { - } \mathrm{HfB}_{2} \text { nanop }
$$

Impregnation
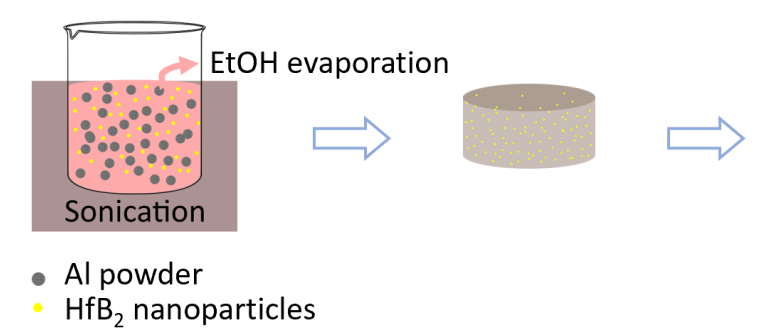

Press into pallet

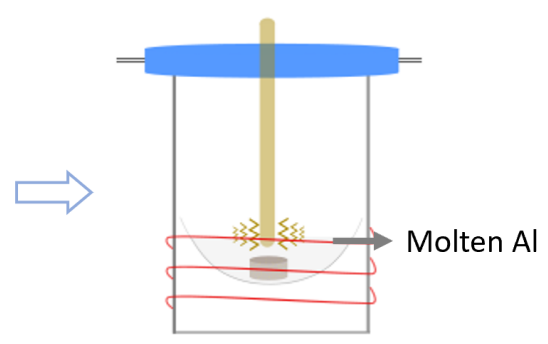

Dilution/Sonication

Figure 1. Scheme of the synthesis procedures of $\mathrm{HfB}_{2}-\mathrm{Al}$ nanocomposite

\section{RESULTS AND DISCUSSION}

\section{$\mathrm{HfB}_{2}$-Al nanocomposites}

$\mathrm{HfB}_{2}$ nanoparticles were prepared by molten salts synthesis. ${ }^{21}$ Different contrasts observed by TEM (Figure 2a) show the core-shell structure of the nanoparticles. TEM shows a diameter of the contrasted core of 4 to $14 \mathrm{~nm}$. This core shows high contrast, which is ascribed to a crystalline character and to a higher electron density, due to the Hf content. ${ }^{21}$ The powder XRD pattern (Figure 2b) is fully indexed along the hexagonal $\mathrm{HfB}_{2}$ structure (S.G: P6/mmm, $\mathrm{a}=0.3149 \mathrm{~nm}, \mathrm{c}=0.3480 \mathrm{~nm})$. The crystallite size of $\mathrm{HfB}_{2}$ calculated according to the Scherrer formula is $8 \mathrm{~nm}$, in agreement with TEM observations, thus confirming that the core is single crystalline and made of $\mathrm{HfB}_{2}$. A less contrasted layer of ca. $3 \mathrm{~nm}$-thick covers the surface of 
$\mathrm{HfB}_{2}$ nanoparticles, corresponding to a boron-rich amorphous layer as described in a previous study. ${ }^{21}$ The diameter measured by dynamic light scattering (DLS) on an aqueous dispersion is about $400 \mathrm{~nm}$, which is larger than the TEM data, indicating the formation of aggregates. These objects are clearly observed by TEM, which indicates that the amorphous shell embeds several particles.
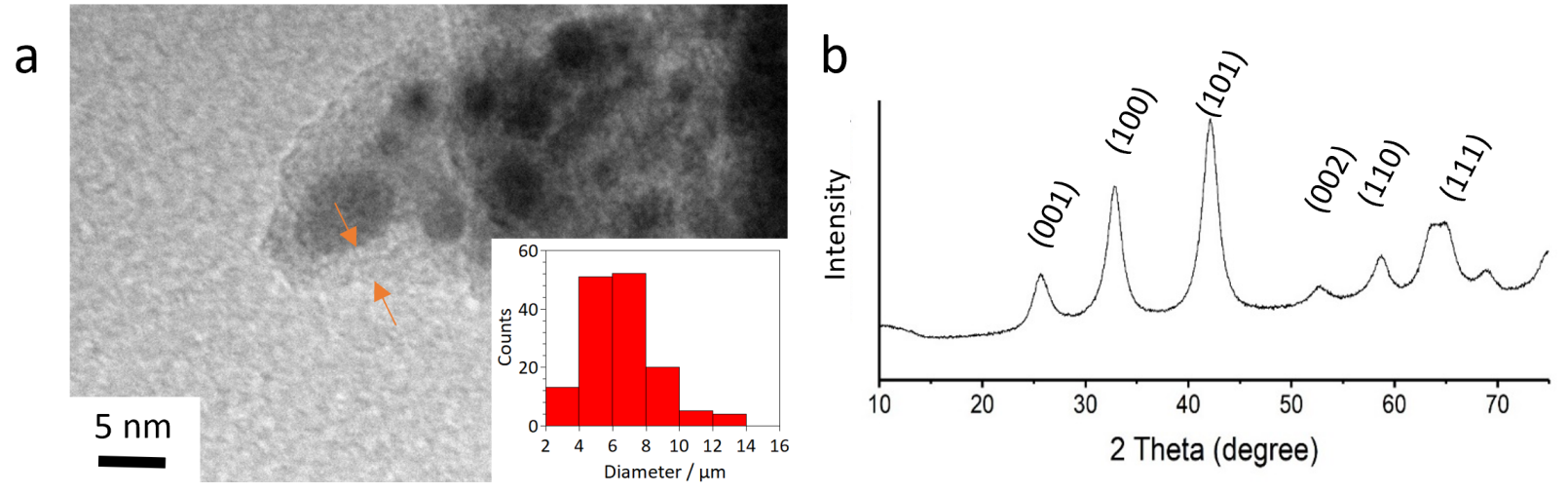

Figure 2. (a) TEM image and (b) powder XRD pattern (indexed along the $\mathrm{HfB}_{2}$ structure) of $\mathrm{HfB}_{2}$ nanoparticles coated with an amorphous boron-rich layer, as shown between two arrows.

0.8 vol.\% $\mathrm{HfB}_{2}-\mathrm{Al}$ nanocomposites were then prepared by ultrasound-assisted dispersion of nanoparticles in molten $\mathrm{Al}$ at $720^{\circ} \mathrm{C}$. To overcome the difficulties of directly loading the nanoparticles powders in the melt, we developed a two-step process to introduce the nanoparticles in the melt (Figure 1): a) dispersing the nanoparticles in ethanol in the presence of a powder of aluminum microparticles, b) pressing the slurry into a pellet, c) diluting the pellet in molten aluminum under ultrasonication followed by liquid nitrogen quenching. Cold pressing of the pellet at room temperature prevents coalescence of the nanoparticles and reactivity between $\mathrm{Al}$ and the boron shell of the nanoparticles that could occur by hot pressing. ${ }^{4}$ The macroscale homogeneity of the as-synthesized nanocomposites was evaluated by wavelength dispersive $\mathrm{X}$ ray fluorescence spectroscopy WDXRF on 3 different cut pieces of the material (Figure S1). 
The chemical composition was identical between all pieces and the inclusion ratio $(\mathrm{Hf} / \mathrm{Al})$ was close to the expected value, showing that the incorporation of the nanoparticles was quantitative.

Broad peaks from XRD patterns (Figure S2) confirmed that the $\mathrm{HfB}_{2}$ structure was maintained. Low intensity peaks at $29^{\circ}$ and $41^{\circ}$ could be indexed along the $\mathrm{AlB}_{12}$ phase, which may arise from the reaction between molten aluminum and the amorphous boron-rich shell of the nanoparticles. These samples were further analyzed by scanning electron microscopy (SEM) coupled to EDS (Figure 3). EDS analyses (Figure 3b-d) show a heterogeneous composition between the boundaries and the grains, which indicates that $\mathrm{HfB}_{2}$ nanoparticles were segregated towards grain boundaries. The average grain size of $\mathrm{Al}$ is $18 \mu \mathrm{m}$, smaller than the average grain size $(27 \mu \mathrm{m})$ of a reference nanocomposite containing 0.8 vol. \% of $35 \mathrm{~nm} \mathrm{SiC} \mathrm{nanoparticles}$ prepared with the same method (Figure S3). Therefore, $\mathrm{HfB}_{2}$ nanoparticles have a clear effect on the matrix grain size by refining the microstructure.
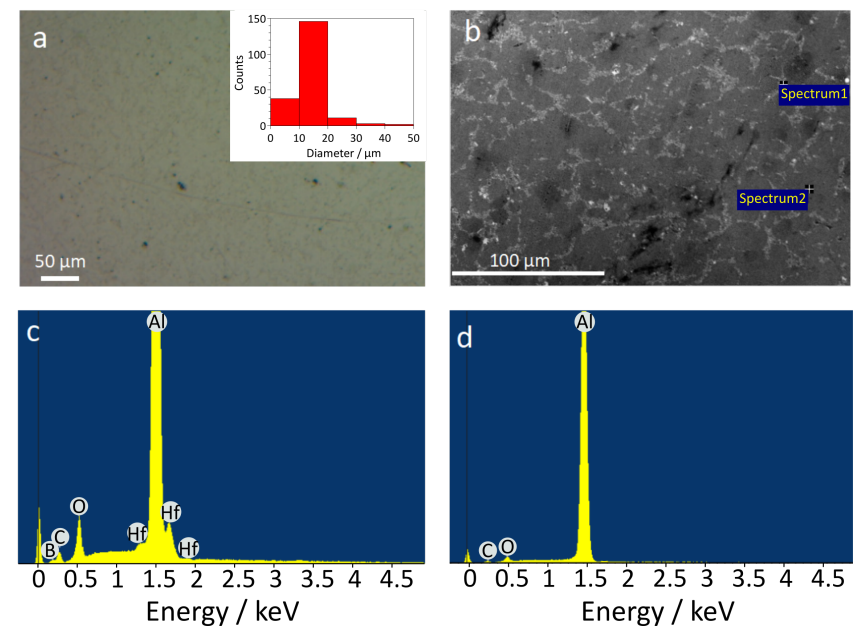

Figure 3. Characterization of a 0.8 vol $\% \mathrm{HfB}_{2}-\mathrm{Al}$ nanocomposite prepared with ultrasound treatment with a $\mathrm{Nb}$ tip for $5 \mathrm{~min}$. and cooled down in liquid nitrogen. (a) Optical image and size distribution of Al grains. (b) backscattered SEM image and corresponding EDS analyses of (c) a 
grain boundary (corresponding to area labelled 1 in b) and of (d) an $\mathrm{Al}$ grain (corresponding to the area labelled 2 in b). Dark and grey contrasts correspond to aluminum and to aggregates of $\mathrm{HfB}_{2}$ nanoparticles, respectively.

TEM images (Figure 4) also show that the nanoparticles formed sub-micrometric aggregates at the Al grain boundaries. No separated nanoparticles could be found in Al grains. Compared to the initial nanoparticle precursors (Figure 2a, b), $\mathrm{HfB}_{2}$ nanoparticles could maintain their size after the harsh melting process at high temperature (Figure 4a-e). The spherelike morphology of the initial particles showed significant faceting in the nanocomposite (Figure 4f). The $\mathrm{HfB}_{2}$ crystal structure was confirmed according to FFT (Figure 4h), also indicating the thermal stability of $\mathrm{HfB}_{2}$ nanoparticles. Some particles exhibit stacking faults (Figure $\mathbf{4 f}$, within white arrows). EDS mapping (Figure 4b-d) confirms co-location of Hf and B elements in the nanoparticles. 

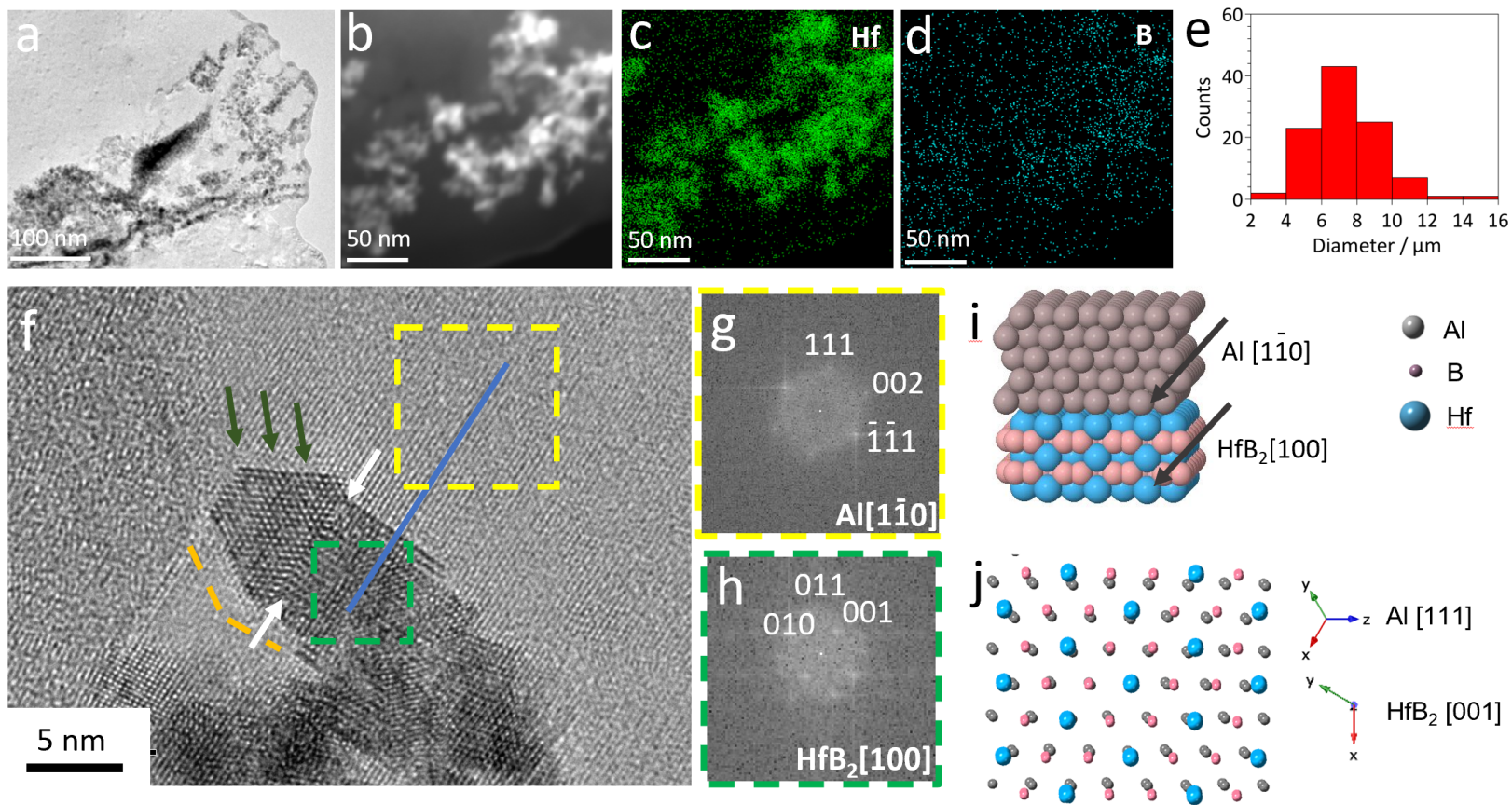

Figure 4. (a) TEM and (b) scanning TEM- high angle annular dark field (STEM-HAADF) images of a 0.8 vol. \% $\mathrm{HfB}_{2}-\mathrm{Al}$ nanocomposite, dark and light parts correspond to $\mathrm{HfB}_{2}$ nanoparticles and to the aluminum matrix, respectively. STEM-EDS mapping showing the overlap of (c) Hf and (d) B in the nanoparticles. (e) Size distribution of the nanoinclusions. (f) HRTEM image. (g, h) FFT patterns of corresponding areas in (f). (i) 3D scheme and (j) normal projection (along $[001]_{\mathrm{HFB} 2}$ and $[111]_{\mathrm{Al}}$ ) of the epitaxial relationship between $\mathrm{Al}$ and $\mathrm{HfB}_{2}$ extracted from the FFT of $(\mathrm{g}, \mathrm{h})$. $\mathrm{Al}$ and $\mathrm{HfB}_{2}$ relate directions in (i) correspond to the zone axes for the two components in (f).

Studying the interface between the aluminum matrix and $\mathrm{HfB}_{2}$ nanoparticles is instrumental in revealing the mechanisms of the nanocomposite formation. ${ }^{22}$ HRTEM at the interface (Figure 4f) indicates three main features: 
a) Epitaxial relationship: $(010)_{\mathrm{HFB} 2} / /(-1-11)_{\mathrm{Al}}$ (blue line in Figure 4f) and $[100]_{\mathrm{HFB} 2} / /[1-$ $10]_{\mathrm{Al}}$. The lattice distance mismatch between $(010)_{\mathrm{HfB} 2}$ and $(-1-11)_{\mathrm{Al}}$ is $11 \%$, which is the smallest that can be found for $\mathrm{HfB}_{2}$. Figures $4 \mathbf{i}$ and $\mathbf{j}$ show the coherent interface between the two phases. This low mismatch is expected to promote epitaxial nucleation of the Al grain during solidification.

b) Distortion of lattice fringes extending up to $1 \mathrm{~nm}$ from the interfaces (green arrows). The mismatch between other $\mathrm{HfB}_{2}$ and $\mathrm{Al}$ lattice planes, except for the plane couple described above, could disturb crystalline order of $\mathrm{Al}$ near the interface, and thus cause $\mathrm{Al}$ lattice distortions to accommodate the $\mathrm{HfB}_{2}$ lattice and achieve energetically favoured coherency. The faceting of the particles after preparation of the composite seems to favour the growth of (001) facets. This supports our hypothesis of heterogeneous nucleation of $\mathrm{Al}$ grains on the $\mathrm{HfB}_{2}$ nanoparticles, which is energetically favoured via the epitaxial relationship observed at the (001) facets and mentioned above. The combination of (a) epitaxial relationship and (b) lattice distortion implies that the $\mathrm{HfB}_{2}-\mathrm{Al}$ interface is semi-coherent, which is expected to enhance the mechanical properties. ${ }^{23}$

c) Few nanometer-thick areas (within orange dashes, Figure 4f) around the particles do not exhibit lattice fringes. Their thickness is consistent with the $c a .2 \mathrm{~nm}$ thick amorphous boronrich layer wrapping the initial nanoparticles. According to the Al-B phase diagram, ${ }^{24} \mathrm{AlB}_{2}$ and $\mathrm{AlB}_{12}$ could form above $500{ }^{\circ} \mathrm{C}$. One may then hypothesize that B-rich $\mathrm{Al}-\mathrm{B}$ compounds could form by reaction between the pristine amorphous boron-rich layer and liquid aluminum during the nanoparticles incorporation, as previously reported for the reaction of liquid aluminum with boron nitride nanotubes after 10 minutes at $650{ }^{\circ} \mathrm{C},{ }^{25}$ conditions similar to those used in our experiments. Because the absence of noticeable lattice fringes may arise from a wrong 
orientation versus the electron beam, we have analysed areas where the Al matrix was away from diffraction conditions, which ensured that structural information was retrieved from the nanoparticles and their surroundings (Figure S4). A $2 \mathrm{~nm}$-thick crystalline layer was observed with lower contrast than the particle's core. HRTEM could be indexed satisfactorily only along the $\mathrm{AlB}_{12}$ structure, in agreement with XRD (Figure S2).

Our observations suggest that although $\mathrm{HfB}_{2}$ nanoparticles segregate at grain boundaries, as reported for other boride- $\mathrm{Al}$ systems,,${ }^{15,26} \mathrm{HfB}_{2}$ nanoparticles can act as efficient heterogeneous nucleation sites during cooling and solidification of $\mathrm{Al}$ grains through two pathways: epitaxial growth of $\mathrm{Al}$ onto $\mathrm{HfB}_{2}$ nanoparticles, or growth of a $\mathrm{B}$-rich $\mathrm{AlB}_{12}$ interphase by reaction between $\mathrm{Al}$ and the B-rich amorphous layer around the initial particles. This would result in enhanced nucleation rate of $\mathrm{Al}$ grains, which would in turn decrease the $\mathrm{Al}$ grain size.

\section{$\mathrm{HfB}_{2}-\mathrm{AlSi}_{7} \mathrm{Mg}_{0.3} \mathrm{Cu}_{0.5}$ nanocomposites}

We have further assessed the possibility to prepare lightweight matrix composites with the $\mathrm{AlSi}_{7} \mathrm{Mg}_{0.3} \mathrm{Cu}_{0.5}$ cast alloy. A pellet consisting of pure $\mathrm{Al}$ and nanoparticles was prepared and incorporated in molten $\mathrm{AlSi}_{7} \mathrm{Mg}_{0.3} \mathrm{Cu}_{0.5}$ at $600{ }^{\circ} \mathrm{C}$. The melting of the pellet took around 5 minutes. Then, ultrasound treatment was applied for 5 minutes with an $\mathrm{Nb}$ tip. The solidification of the material was carried out by quenching in liquid nitrogen. Optical microscopy (Figure 5a) highlights the Si phase in dark grey. SEM-EDS mapping (Figure 5c-f) suggests that hafnium is located at the Si-rich grain boundaries, in agreement with the results obtained with the Al matrix. 
We then investigated the effects of the nanoparticles on the structure of the metal matrix during solidification. A reference sample was obtained by incorporating a pure aluminum pellet to $\mathrm{AlSi}_{7} \mathrm{Mg}_{0.3} \mathrm{Cu}_{0.5}$ alloy, followed by identical ultrasound treatment and cooling protocol (Figure S5). Compared to pristine $\mathrm{AlSi}_{7} \mathrm{Mg}_{0.3} \mathrm{Cu}_{0.5}$ (Figure S6), the sample treated with ultrasounds (Figure S5) exhibits a globular grain structure, in accordance with previous reports. ${ }^{2,27,28}$ The microstructure of the alloy was refined by incorporation of the particles, with the $\mathrm{Al}$ grain size decreasing from $78 \mu \mathrm{m}$ (Figure S5) to $43 \mu \mathrm{m}$ (Figure 5). This grain refinement effect is less marked than with $\mathrm{HfB}_{2}-\mathrm{Al}$ samples. This may be ascribed to the alloy solute responsible of constitutional undercooling during solidification, which plays a primary role in the grain size.
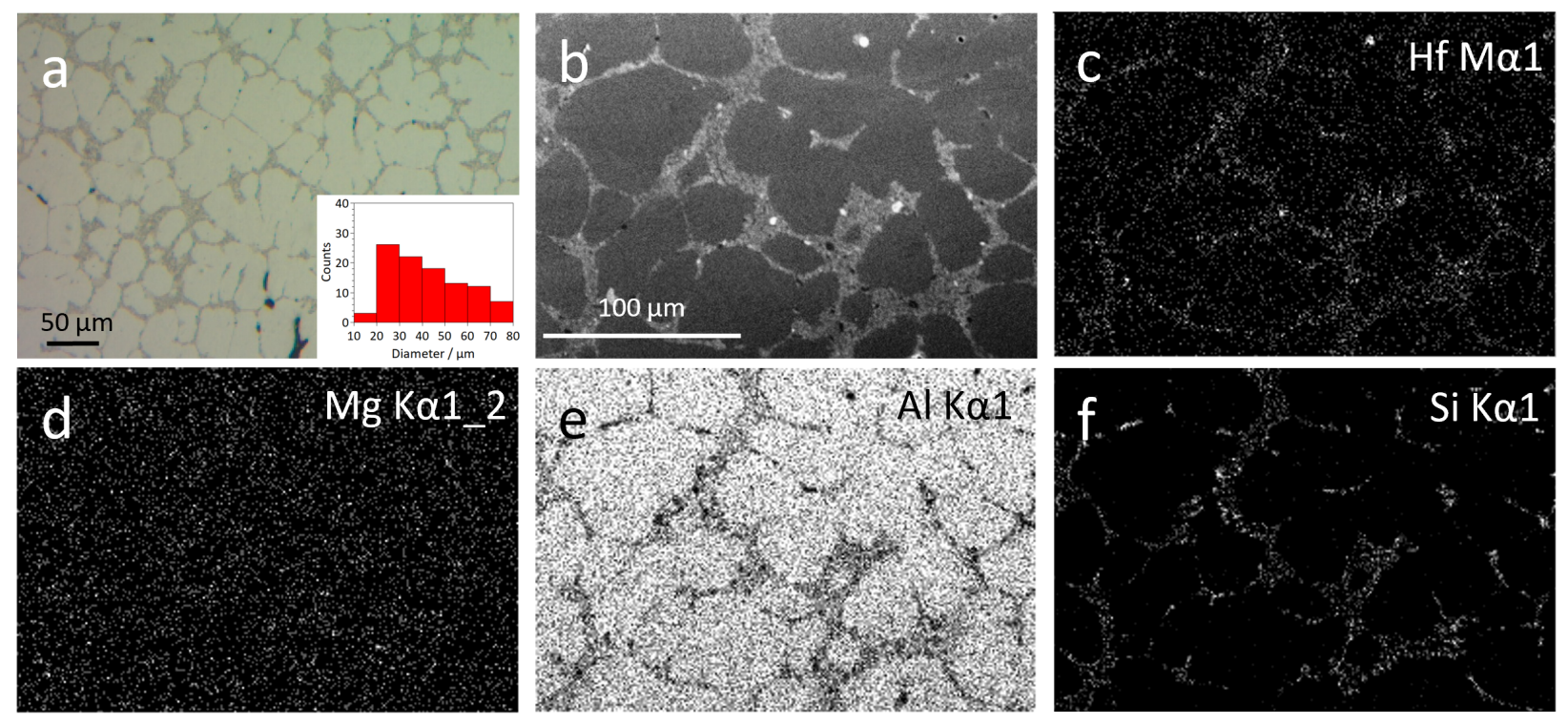

Figure 5. Microscopy study of a 0.8 vol\% $\mathrm{HfB}_{2}-\mathrm{AlSi}_{7} \mathrm{Mg}_{0.3} \mathrm{Cu}_{0.5}$ nanocomposite prepared by ultrasound treatment with a $\mathrm{Nb}$ tip for 5 minutes and cooled down in liquid nitrogen. (a) Optical microscopy image and corresponding size distribution of Al grains, (b) SEM micrograph and (cf) corresponding SEM-EDS maps. The scales are identical from (b) to (f). 


\section{Hardness properties}

The mechanical properties were firstly investigated by microhardness measurements on several areas. For nanocomposites containing 0.8 vol. $\%$ of $\mathrm{HfB}_{2}$ nanoparticles, the measured hardness (Table 1) of $33 \mathrm{HV}$ was larger than the hardness of sole aluminum (24 HV) prepared by the same way. The $38 \%$ hardness increase is attributed to the hard nanoparticles at the grain boundaries. In the case of the cast alloy prepared by a similar way, the reference sample incorporating additional $\mathrm{Al}$ with a mass ratio alloy: $\mathrm{Al}$ of 1:1 showed a lower hardness (67.2 HV) than the bare alloy $(97.9 \mathrm{HV})$. When additional $\mathrm{Al}$ was incorporated together with $\mathrm{HfB}_{2}$ nanoparticles, the hardness hardly changed $(71.7 \mathrm{HV})$. This result is consistent with microscopy observations, suggesting the Si-based solute of the alloy segregates at grain boundaries and is responsible for the hardness properties.

Table 1. Microhardness of $\mathrm{HfB}_{2}-\mathrm{Al}$ nanocomposites

\begin{tabular}{|c|c|c|c|c|}
\hline Sample & $\begin{array}{c}\text { Average hardness } \\
(\mathrm{HV} 0.05)\end{array}$ & $\begin{array}{l}\text { Average Al grains } \\
\text { (HV 0.05) }\end{array}$ & $\begin{array}{c}\text { Average grain } \\
\text { boundaries (HV 0.05) }\end{array}$ & $\begin{array}{c}\text { standard deviation } \\
\text { (HV 0.05) }\end{array}$ \\
\hline Al & 24.9 & l & l & 1.3 \\
\hline $0.8 \mathrm{vol}^{2} \mathrm{HfB}_{2}-\mathrm{Al}$ & 34.9 & 29.2 & 39.4 & 5.0 \\
\hline $\mathrm{AlSi}_{7} \mathrm{Mg}_{0.5} \mathrm{Cu}_{0.5}$ & 97.9 & 80.7 & 126.8 & 16.5 \\
\hline Al-AlSi ${ }_{7} \mathrm{Mg}_{0.5} \mathrm{Cu}_{0.5}$ & 67.2 & 48.1 & 80.7 & 9.8 \\
\hline $\begin{array}{c}0.8 \mathrm{vol} \% \mathrm{HfB}_{2}-\mathrm{Al}- \\
\mathrm{AlSi}_{7} \mathrm{Mg}_{0.5} \mathrm{Cu}_{0.5}\end{array}$ & 71.7 & 51.7 & 91.6 & 12.8 \\
\hline
\end{tabular}

\footnotetext{
* HV 0.05: micro vickers hardness measured with a load of $0.05 \mathrm{kgf}$.
} 
We then used nanoindentation to assess the influence of sub-microscopic heterogeneities on hardness and on the elastic modulus. The mechanical properties of wide areas containing multiple grains and grain boundaries (optical microscopy image in Figure 6a) were mapped with indents depths of $3 \mu \mathrm{m}$ every $30 \mu \mathrm{m}$ (Table S1) and of $1 \mu \mathrm{m}$ every $10 \mu \mathrm{m}$ (Table 2, Figure $6 \mathbf{b}$ and c). For the 0.8 vol. $\% \mathrm{HfB}_{2}-\mathrm{Al}$ nanocomposite (Figure 6, Table S1 and Table 2), a bimodal distribution of hardness is observed: a first population with a relatively low hardness corresponding to the grains and a second population with significantly higher values at grain boundaries, in agreement with the localization of the nanoparticles of hard $\mathrm{HfB}_{2}$. Elastic modulus values show the same trend (Figure 6c). Similar observations were made for a $\mathrm{HfB}_{2}-$ $\mathrm{AlSi}_{7} \mathrm{Cu}_{0.5} \mathrm{Mg}_{0.3}$ nanocomposite (Figure S7).
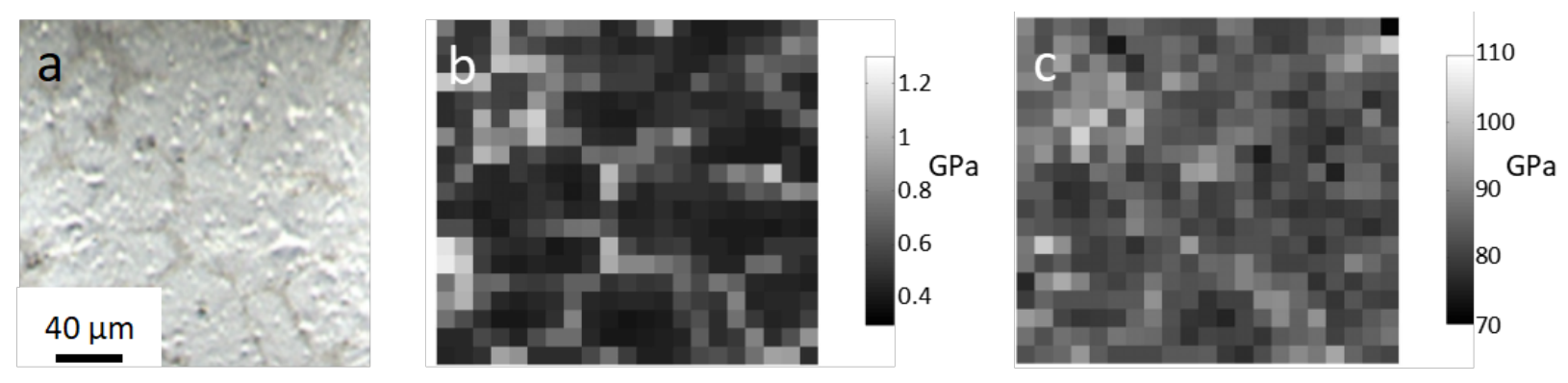

Figure 6. (a) Optical microscopy image, corresponding (b) hardness and (c) elastic modulus of a $0.8 \% \mathrm{HfB}_{2}-\mathrm{Al}$ nanocomposite measured with nanoindentation, $1 \mu \mathrm{m}$ indents. Black pixels at 0 $\mathrm{GPa}$ in (b) and (c) correspond to micropores ascribed to the removal of nanoparticles during polishing.

Table 2. Elastic modulus from nanoindentation (at a depth of $1 \mu \mathrm{m}$ ). 


\begin{tabular}{|c|c|c|c|c|c|c|c|c|c|}
\hline \multirow[t]{2}{*}{ Matrix } & \multicolumn{3}{|c|}{ Hardness (GPa) } & \multicolumn{3}{|c|}{$\begin{array}{c}\text { Hardness of central } \\
\text { population }(\mathrm{GPa})\end{array}$} & \multicolumn{3}{|c|}{$\begin{array}{c}\text { Elastic modulus of central } \\
\text { population }(\mathrm{GPa})\end{array}$} \\
\hline & Average & SD & Median & Average & SD & Median & Average & SD & Median \\
\hline $\mathrm{Al}$ & 0.47 & 0.05 & 0.45 & 0.45 & 0.02 & 0.46 & 80 & 1.3 & 79 \\
\hline $0.8 \mathrm{vol} \% \mathrm{HfB}_{2}-\mathrm{Al}$ & 0.58 & 0.17 & 0.51 & 0.54 & 0.09 & 0.51 & 84 & 2.7 & 84 \\
\hline Al-AlSi ${ }_{7} \mathrm{Mg}_{0.5} \mathrm{Cu}_{0.5}$ & 1.00 & 0.23 & 0.90 & 0.95 & 0.12 & 0.92 & 90 & 2 & 90 \\
\hline $\begin{array}{l}0.8 \mathrm{vol}_{\%} \mathrm{HfB}_{2} \text {-Al- } \\
\mathrm{AlSi}_{7} \mathrm{Mg}_{0.5} \mathrm{Cu}_{0.5}\end{array}$ & 1.15 & 0.34 & 0.94 & 1.02 & 0.24 & 0.95 & 90 & 4 & 89 \\
\hline
\end{tabular}

SD stands for standard deviation

The central population eliminates the $25 \%$ lowest values and the $25 \%$ highest values

$\mathrm{HfB}_{2}$ shows reinforcement of hardness properties compared to bare aluminum with an improvement of 23\% for (Table S1 and Table 2). The cumulative distribution curves with 400 measurements (Figure 7) show a statistical assessment of mechanical properties. For low hardness regions, the plateau for 0.8 vol. $\% \mathrm{HfB}_{2}-\mathrm{Al}$ was similar to pure $\mathrm{Al}$ at around $0.45 \mathrm{GPa}$, confirming that the grains are mostly made of $\mathrm{Al}$ and that most $\mathrm{HfB}_{2}$ nanoparticles were segregated towards grain boundaries. The distribution curve for the $\mathrm{HfB}_{2}-\mathrm{Al}$ composite deviates from the behavior of sole aluminum towards higher hardness, for more than $50 \%$ of the measurements (from 200 to 400), which corresponds to reinforced area at grain boundaries according to mapping (Figure 6). The average of this population calculated from the highest 200 measurements is $0.66 \mathrm{GPa}$, clearly above the values for pure $\mathrm{Al}$, confirming the hardness of $\mathrm{HfB}_{2}$ nanoparticle and their efficient role as grain boundary reinforcements. 

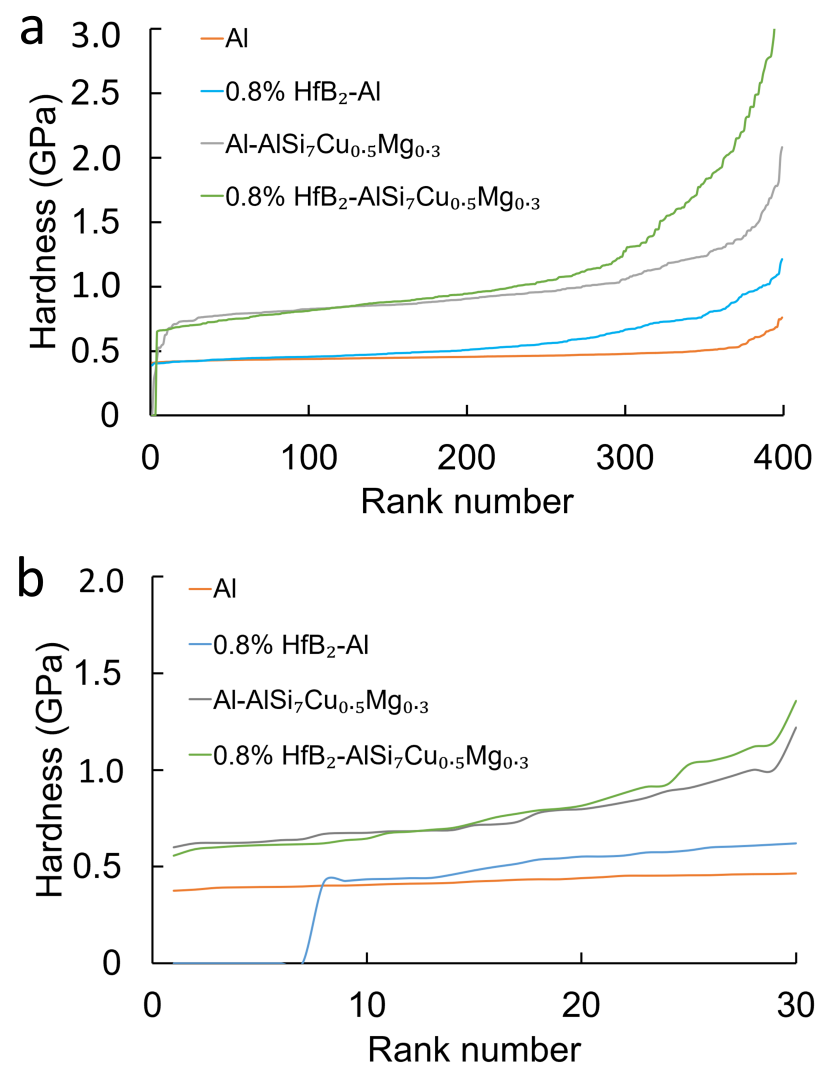

Figure 7. Statistic evaluation of hardness measured at $1 \mu \mathrm{m}$ (a) and $3 \mu \mathrm{m}$ (b) for reference Al and $\mathrm{Al}-\mathrm{AlSi}_{7} \mathrm{Mg}_{0.3} \mathrm{Cu}_{0.5}$ samples, for the 0.8 vol. $\% \mathrm{HfB}_{2}-\mathrm{Al}$ and $\mathrm{HfB}_{2}-\mathrm{Al}-\mathrm{AlSi}_{7} \mathrm{Mg}_{0.3} \mathrm{Cu}_{0.5}$ nanocomposites.

In the case of the $\mathrm{AlSi}_{7} \mathrm{Mg}_{0.3} \mathrm{Cu}_{0.5}$ cast alloy, a similar hardness has been measured between nanocomposites and reference sample (Table $\mathbf{S 1}$ and 2). The large standard deviation of $0.2 \mathrm{GPa}$ is mainly related to the different hardness values of $\mathrm{Al}$ grains and of Si-rich grain boundary regions. The distribution curves (Figure 7) show a similar trend as the the Al-based materials: overlapping of the curves for the the bare matrix and for the composite for the 240 lowest hardness values, corresponding to the grains, and a deviation towards higher hardness values is observed for the highest 160 values in the presence of $\mathrm{HfB}_{2}$ nanoparticles. The tangential midpoint of the distribution curves between the $250^{\text {th }}$ and $400^{\text {th }}$ measurements are 1.8 
$\mathrm{GPa}$ for the sample with nanoparticles and $1.4 \mathrm{GPa}$ for the reference matrix. Therefore, $\mathrm{HfB}_{2}$ nanoparticles enhances the hardness by $29 \%$, again at the grain boundaries (Figure S7).

The elastic modulus has also been measured by nanoindentation (Figure 6c, Table S1 and 2.). The incorporation of $0.8 \%$ nanoparticles of $\mathrm{HfB}_{2}$ in aluminum improve the average elastic modulus by $1.4 \%$ according to measurements with $1 \mu \mathrm{m}$ indents. On contrary, deeper indents of $3 \mu \mathrm{m}$ do not exhibit a significant increase of the modulus compared to the $\mathrm{Al}$ reference, which is attributed to the volume averaging of the measurements with deeper indents.

\section{CONCLUSIONS}

By using ultrasound-assisted casting, we successfully developed new Al matrix nanocomposites containing $\mathrm{HfB}_{2}$ nanoparticles synthesized in molten salts. These nanoparticles are stable in the conditions of liquid metallurgy and segregated at the grain boundaries during solidification of the composite. Detailed examination of the interface between the nanoparticles and the Al matrix revealed first the surface reconstruction of the nanoparticles that showed strong faceting, second an epitaxial relationship between the matrix and the particles, third the likely crystallization of a B-rich boride interphase. We hypothesize that these structural features strongly impact the nucleation of the matrix grains during solidification, where the nanoparticles could act as seeds for the heterogeneous nucleation of aluminum grains. This in turn, has a strong impact on the microstructure of the nanocomposite, which exhibits a significant decrease of the grain size and then an increase in the density of grain boundaries. Sub-microscale mapping of mechanical properties shows how the heterogeneous microstructure reflects local heterogeneous mechanical behavior, with low hardness matrix grains and high hardness grain boundaries where the 
nanoparticles of hard boride are concentrated. These results suggest that further control of the surface states of the pristine nanoparticles, especially their dispersion, the nature and the thickness of their amorphous shell will be relevant parameters to tune the dispersion of boride particles towards original aluminum-based nanocomposites. 


\section{EXPERIMENTAL METHODS}

Preparation of boride nanoparticles. $\mathrm{HfB}_{2}$ nanoparticles were prepared in molten salts as previously reported..$^{21} 0.5 \mathrm{mmol}$ of hafnium (IV) chloride, $1 \mathrm{mmol}$ of sodium borohydride and $2.5 \mathrm{~g}$ of eutectic mixture $\mathrm{LiCl} / \mathrm{KCl}$ powders were ground together with a Retsch MM400 ball miller for 2 minutes at $20 \mathrm{~Hz}$. The reaction medium was heated in a glassy carbon crucible under argon flow at $900{ }^{\circ} \mathrm{C}$ for $4 \mathrm{~h}$. After cooling down to room temperature, the nanoparticles were washed with 12 cycles of centrifugation-redispersion in water and dried under vacuum over night.

Preparation of nanocomposites. $2 \mathrm{~g}$ of aluminum powders (325 mesh, 99.99\%) were added to a suspension of $60 \mathrm{mg}$ of nanoparticle powders in $2 \mathrm{~mL}$ of ethanol, dispersed by sonication at $80{ }^{\circ} \mathrm{C}$ as set point for 40 minutes. Then the suspension was dried under vacuum at $40{ }^{\circ} \mathrm{C}$ for $1 \mathrm{~h}$, followed by pressing into a pellet under 8 tons for $2 \mathrm{~min}$. The pellet and $1 \mathrm{~g}$ of pure $\mathrm{Al}$ beads were then heated by an induction oven at $720{ }^{\circ} \mathrm{C}$ under argon flow in an alumina crucible (Figure S8). After dwelling for $1 \mathrm{~min}$, ultrasounds (80 W, QSonica, Q500A-220, USA) were applied through a sonication horn (4 mm diameter niobium tip) for $5 \mathrm{~min}$., with an interval of $5 \mathrm{~s}$ on / $5 \mathrm{~s}$ off. The final composites were obtained by liquid nitrogen quenching. They were then cut with a diamond wire saw for further analyses.

Powder X-ray diffraction (XRD) was performed with a D8 Bruker diffractometer operating with $\mathrm{Cu} \mathrm{K}$ radiation in Bragg-Brentano configuration. The ICSD reference card 30422 was used for the indexation of the $\mathrm{HfB}_{2}$ structure. The COD reference card 9012429 was used for the 
indexation of $\mathrm{Al}$. The COD reference card 1527618 was used for the indexation of the $\alpha-\mathrm{HfB}_{12}$ structure.

Transmission electron microscopy (TEM). The nanoparticles were dispersed in ethanol, then one drop was evaporated on carbon-coated copper grids. For the prepared composites, thin foils were prepared as $3 \times 3 \times 0.5 \mathrm{~mm}$ rectangles cut in the middle of the samples. The polishing step was then performed with a diamond disk to get a $200 \mu \mathrm{m}$ thick sample and the thickness of the centre was further reduced to $50 \mu \mathrm{m}$ by using a precision dimpling instrument (Model 515, South Bay Technology). Ion milling was performed for final thinning. TEM images were recorded on a Tecnai spirit G2 microscope operating at 120kV. The STEM images and STEM-EDX mapping were recorded with a JEM 2100Plus UHR microscope, and were both performed at $200 \mathrm{kV}$. The size distribution of the nanoparticles was determined by counting 120 to 200 nanoparticles from TEM images. The interface relationship was simulated with the software Eje-Z and Rhodius. The size of the supercell used to build the model was 20x20x20 unit cells.

Scanning electron microscopy (SEM). The samples were mounted in polyfast resin (Struers), followed by a series of polishing steps through 1000, 2400, 4800 grit papers (Buehler), then with $3 \mu \mathrm{m}$ and $1 \mu \mathrm{m}$ diamond suspensions. The samples were directly attached on a SEM holder using carbon tape. They were then observed under scanning electron microscopy (SEM, Hitachi S-3400N and Hitachi SU-70 microscope) equipped with an Oxford X-Max 50 mm2 EDS detector. Image analysis was performed with the Fiji software. The grain size distribution was measured by ImageJ from optical microscope images and SEM images, by counting the area of over 120 cells which boundary was defined by the inclusion aggregates. 
Dynamic light scattering (DLS). The average hydrodynamic diameter of $\mathrm{HfB}_{2}$ aggregates dispersed in water was determined with a Zetasizer Nano ZS90 apparatus (Malvern Panalytical).

The Vickers hardness was measured with a load of $0.05 \mathrm{~kg}$, dwell time $10 \mathrm{~s}$ using Wilson VH1102 microhardness testers.

The nanoindentation measurements were realized using a Nano Indenter G200 from Agilent equipped with a Berkovich tip. The hardness was measured at the unloading process using Oliver and Pharr method. 399 measurements were achieved on a matrix of $21 * 19$ with a distance of 10 $\mu \mathrm{m}$ at a depth of $1 \mu \mathrm{m}$ to obtain a mapping of mechanical properties. A matrix of $6 * 5$ with a depth of $3 \mu \mathrm{m}$ was also measured to obtain an average measurement of mechanical properties.

\section{Supporting Information.}

Figures S1 to S9.

\section{Author Contributions}

B.M., S.D., C.S. and D.P. conceived the concept. B.M. performed synthesis, fabrication, SEM and EDS characterization. L.M. prepared the TEM samples. BM and I.G.R. performed TEM characterization and analysis. I.G.R. performed the interface simulation. P-E.M. performed nanoindentation experiments and analyzed the results. C.S., S.D. and D.P supervised the project.

\section{ACKNOWLEDGMENT}


The authors would like to thank the funding by Stellantis and ANRT and the framework of the OpenLab $\beta \chi \Phi$ involving Stellantis and Sorbonne University. 


\section{REFERENCES}

(1) Ibrahim, I. A.; Mohamed, F. A.; Lavernia, E. J. Particulate Reinforced Metal Matrix Composites - a Review. J. Mater. Sci. 1991, 26 (5), 1137-1156.

(2) Casati, R.; Vedani, M. Metal Matrix Composites Reinforced by Nano-Particles-A Review. 2014, 65-83.

(3) Mohanty, P.; Mahapatra, R.; Padhi, P.; Ramana, C. H. V. V.; Mishra, D. K. Ultrasonic Cavitation: An Approach to Synthesize Uniformly Dispersed Metal Matrix Nanocomposites-A Review. Nano-Structures and Nano-Objects 2020, 23, 100475.

(4) Estruga, M.; Chen, L.; Choi, H.; Li, X.; Jin, S. Ultrasonic-Assisted Synthesis of SurfaceClean $\mathrm{TiB}_{2}$ Nanoparticles and Their Improved Dispersion and Capture in Al-Matrix Nanocomposites. ACS Appl. Mater. Interfaces 2013, 5 (17), 8813-8819.

(5) Malaki, M.; Xu, W.; Kasar, A. K.; Menezes, P. L.; Dieringa, H. Advanced Metal Matrix Nanocomposites.

(6) Hsu, C. J.; Chang, C. Y.; Kao, P. W.; Ho, N. J.; Chang, C. P. Al-Al3Ti Nanocomposites Produced in Situ by Friction Stir Processing. Acta Mater. 2006, 54 (19), 5241-5249.

(7) Cao, C.; Ling, H.; Murali, N.; Li, X. In-Situ Molten Salt Reaction and Incorporation of Small (10 Nm) TiC Nanoparticles into Al. Materialia 2019, 7.

(8) Liu, X.; Liu, Y.; Huang, D.; Han, Q.; Wang, X. Tailoring In-Situ TiB 2 Particulates in Aluminum Matrix Composites. Mater. Sci. Eng. A 2017, 705 (May), 55-61.

(9) Liu, W.; Cao, C.; Xu, J.; Wang, X.; Li, X. Molten Salt Assisted Solidification Nanoprocessing of Al-TiC Nanocomposites. Mater. Lett. 2016, 185 (September), 392395.

(10) Nazari, M.; Eskandari, H.; Khodabakhshi, F. Production and Characterization of an Advanced AA6061-Graphene-TiB 2 Hybrid Surface Nanocomposite by Multi-Pass Friction Stir Processing. Surf. Coatings Technol. 2019, 377.

(11) Pan, S.; Yuan, J.; Zhang, P.; Sokoluk, M.; Yao, G.; Li, X. Effect of Electron Concentration on Electrical Conductivity in in Situ Al-TiB 2 Nanocomposites. Appl. Phys. Lett. 2020, 116 (1).

(12) Portehault, D.; Delacroix, S.; Gouget, G.; Grosjean, R.; Chan-Chang, T. H. C. Beyond the Compositional Threshold of Nanoparticle-Based Materials. Acc. Chem. Res. 2018, 51 (4), 930-939.

(13) Gupta, S. K.; Mao, Y. Recent Developments on Molten Salt Synthesis of Inorganic 
Nanomaterials : A Review. 2021.

(14) Li, M.; Ma, K.; Jiang, L.; Yang, H.; Lavernia, E. J.; Zhang, L.; Julie, M. Synthesis and Mechanical Behavior of Nanostructured Al 5083/ n-TiB 2 Metal Matrix Composites. Mater. Sci. Eng. A 2016.

(15) Schaffer, P. L.; Miller, D. N.; Dahle, A. K. Crystallography of Engulfed and Pushed TiB 2 Particles in Aluminium. Scr. Mater. 2007, 57 (12), 1129-1132.

(16) Xie, C.; Zhang, Q.; Zakaryan, H. A.; Wan, H.; Liu, N.; Kvashnin, A. G.; Oganov, A. R. Stable and Hard Hafnium Borides: A First-Principles Study. J. Appl. Phys. 2019, 125 (20).

(17) Kasraee, K.; Tayebifard, S. A.; Roghani, H.; Shahedi Asl, M. Preparation of B ${ }_{4} \mathrm{C}-\mathrm{SiC}-$ $\mathrm{HfB}_{2}$ Nanocomposite by Mechanically Activated Combustion Synthesis. Ceram. Int. 2020, 46 (8), 12288-12295.

(18) Dhandapani, S.; Rajmohan, T.; Palanikumar, K.; Charan, M. Synthesis and Characterization of Dual Particle $\left(\mathrm{MWCT}+\mathrm{B}_{4} \mathrm{C}\right)$ Reinforced Sintered Hybrid Aluminum Matrix Composites. Part. Sci. Technol. 2016, 34 (3), 255-262.

(19) Sairam, K.; Sonber, J. K.; Murthy, T. S. R. C.; Subramanian, C.; Hubli, R. C.; Suri, A. K. Development of $\mathrm{B}_{4} \mathrm{C}-\mathrm{HfB}_{2}$ Composites by Reaction Hot Pressing. Int. J. Refract. Met. Hard Mater. 2012, 35, 32-40.

(20) Li, P.; Zhou, R.; Zeng, X. C. Computational Analysis of Stable Hard Structures in the TiB System. ACS Appl. Mater. Interfaces 2015, 7 (28), 15607-15617.

(21) Portehault, D.; Devi, S.; Beaunier, P.; Gervais, C.; Giordano, C.; Sanchez, C.; Antonietti, M. A General Solution Route toward Metal Boride Nanocrystals. Angew. Chemie 2011, 123 (14), 3320-3323.

(22) Easton, M. A. D. S. J. Grain Refinement of Aluminum Alloys: Part I. The Nucleant and Solute Paradigms. Metall. Mater. Trans. 1999, 30 A (6), 1613.

(23) Luo, Z. P. Crystallography of $\mathrm{SiC} / \mathrm{MgAl}_{2} \mathrm{O}_{4} / \mathrm{Al}$ Interfaces in a Pre-Oxidized $\mathrm{SiC}$ Reinforced SiC/Al Composite. Acta Mater. 2006, 54 (1), 47-58.

(24) Wang, X. The Formation of $\mathrm{AlB}_{2}$ in an Al-B Master Alloy. J. Alloy. Comp. 2005, 403, 283-287.

(25) Nautiyal, P.; Gupta, A.; Seal, S.; Boesl, B.; Agarwal, A. Reactive Wetting and Filling of Boron Nitride Nanotubes by Molten Aluminum during Equilibrium Solidification. Acta Mater. 2017, 126, 124-131.

(26) Firestein, K. L.; Corthay, S.; Steinman, A. E.; Matveev, A. T.; Kovalskii, A. M.; Sukhorukova, I. V. High-Strength Aluminum-Based Composites Reinforced with BN, 
AlB2 and AlN Particles Fabricated via Reactive Spark Plasma Sintering of Al-BN Powder Mixtures. Mater. Sci. Eng. A 2017, 681 (November 2016), 1-9.

(27) Prasad Reddy, A.; Vamsi Krishna, P.; Narasimha Rao, R.; Murthy, N. V. Silicon Carbide Reinforced Aluminium Metal Matrix Nano Composites-A Review. Mater. Today Proc. 2017, 4 (2), 3959-3971.

(28) Liang, G.; Ali, Y.; You, G.; Zhang, M. X. Effect of Cooling Rate on Grain Refinement of Cast Aluminium Alloys. Materialia 2018, 3 (June), 113-121.

(29) Chen, L. Y.; Xu, J. Q.; Choi, H.; Pozuelo, M.; Ma, X.; Bhowmick, S.; Yang, J. M.; Mathaudhu, S.; Li, X. C. Processing and Properties of Magnesium Containing a Dense Uniform Dispersion of Nanoparticles. Nature 2015, 538 (7583), 539-543.

(30) Klement W, Willens RH, D. P. No.; Non-Crystalline Structure in Solidified Gold-Silicon Alloys. Itle. Nature 1960, 187(4740), 869-870. 